\title{
Identifying Efficient Exchange Rate Dynamics from Noisy Data
}

\author{
$\underline{\text { Felix Chan }}^{\text {a }}$ \\ ${ }^{a}$ School of Economics and Finance, Curtin University, GPO BOX U1987, Perth, Western Australia, 6845 \\ Email: F.Chan@curtin.edu.au
}

\begin{abstract}
This paper proposes a new methodology to estimate a simple spot and forward exchange rates model. The method is inspired by the recent development in Independent Component Analysis (ICA) and it allows the identification and estimation of efficient exchange rate (exchange rate when market is efficient) and external market influences (market noise).

Consider the linear equation $\mathbf{y}_{t}=A \mathbf{x}_{t}$, where $\mathbf{y}_{t}$ and $\mathbf{x}_{t}$ are $k \times 1$ vectors of observable and unobservable random variables, respectively, and $A$ is a $k \times k$ matrix. Under the assumption that each element in $\mathbf{x}_{t}$ is independent to each other and $\mathbf{x}_{t}$ consists of no more than one normal variate, then Independent Component Analysis (ICA) provides a convenient framework to recover the mixing matrix $A$, subject to scaling and permutation, by utilising the independence and non-normality nature of $\mathbf{x}_{t}$. Subsequently, it is also possible to recover $\mathbf{x}_{t}$ based on the observations of $\mathbf{y}_{t}$, subject to scaling and permutation.
\end{abstract}

Let $\mathbf{y}_{t}$ denotes a $k \times 1$ vector of co-integrated $I(1)$ variables, then following the Granger's Representation Theorem and the Phillips' Triangular Representation, there exists a $k \times k$ matrix, $A$, and a $k \times 1$ vector, $\mathbf{x}_{t}$, such that $\mathbf{y}_{t}=A \mathbf{x}_{t}$. Moreover, there are exactly $r I(0)$ elements and $k-r I(1)$ elements in $\mathbf{x}_{t}$. This paper shows that, under the same assumptions of ICA, it is possible to estimate $A$, and recover the unobserved random variables, $\mathbf{x}_{t}$, based solely on the observations of $\mathbf{y}_{t}$. In order words, this paper proposes a new test of co-cointegration based on ICA. This is particularly useful as standard co-integration analysis assumes normality which is unlikely to be true for most high frequency financial time series. Thus, the proposed technique is particularly suitable for analysing high frequency financial time series data, such as stock prices and exchange rates.

The paper then proposes a simple model of spot and forward exchange rates which assumes that both rates are linear combinations of two unobserved components, namely, efficient exchange rate and market noise. The paper shows that the proposed co-integration test can be applied to the model in order to differentiate the efficient exchange rate and the market noise.

This paper applies the proposed method to the daily US/Australia spot and forward exchange rates. By analysing the dynamics in the efficient rates and market noise, this paper obtains evidence against some of the standard assumptions underlying conventional exchange rate models and market micro-structure noise.

Keywords: Blind source separation, Independent component analysis, Cointegration rank, Efficient exchange rate 


\section{INTRODUCTION}

This paper proposes a new methodology to identify efficient exchange rate (exchange rate when market is efficient) and market noise from daily data. The methodology is inspired by the recent development in Independent Component Analysis (ICA) and the paper has two main contributions. Firstly, this paper proposes a new method of identifying cointegrating relationships between a set of $I(1)$ variables that are non-normally distributed. Secondly, the paper proposes a simple model of spot and forward rates, so that the efficient exchange rate and the market noise can be identified by applying the proposed method of cointegration.

The forward-spot relationship has been studied extensively in the literature, see for examples, Mark (1990), Naka and Whitney (1995), Liuntel and Paudyal (1998) and Zivot (2000). Let $s_{t}$ and $f_{t}$ denote the logarithms of spot and forward exchange rates at time $t$, respectively, then the forward rate unbiasedness hypothesis (FRUH) suggests that $s_{t+1}$ and $f_{t}$ should be cointegrated with the cointegrating vector $(1,-1)^{\prime}$. Zivot (2000) argued that if $s_{t}-f_{t}$ is stationary then $s_{t+1}-f_{t}$ must also be stationary but the underlying Vector Error Correction Model (VECM) will be much more complicated. Therefore, inference based on a simple VECM model for $s_{t+1}$ and $f_{t}$ may not be valid. More importantly, testing FRUH using high frequency data with existing cointegration techniques may be problematic for at least two reasons. Firstly, most daily financial time series have high excess kurtosis and hence, the assumption of normality does not hold. Secondly, the conditional variance of most high frequency financial time series are time-varying and its impact on testing co-integrating relationship is still unclear. This paper proposes a new test of cointegration and a simple model of spot and forward exchange rates that will address these issues.

The paper is organised as follows: Section 2 will present a simple model of spot-forward exchange rates. This is followed by an introduction of the new cointegration test in Section 3. Empirical results can be found in Section 4 and the Section 5 contains concluding remarks.

\section{MODEL}

Consider the following model of spot and forward rates:

$$
\left(\begin{array}{c}
s_{t} \\
f_{t}
\end{array}\right)=A\left(\begin{array}{c}
e_{t} \\
n_{t}
\end{array}\right)
$$

where $s_{t}$ and $f_{t}$ denote the logarithm of spot and forward rates, respectively. $A=\left\{a_{i j}\right\}$ for $i, j=1,2$ is a $2 \times 2$ matrix with full rank. $e_{t}$ and $n_{t}$ denote the efficient exchange rate and market noise, respectively. This model has two implications under the assumption that the market noise is stationary with zero mean. Firstly, the non-stationary nature of both spot and forward rates comes from the efficient exchange rate, $e_{t}$. Secondly, the market noise, $n_{t}$, represents the deviation from the equilibrium. To see this, let $|A|$ denotes the determinant of $A$ and invert equation (1) yields:

$$
\frac{1}{|A|}\left(\begin{array}{cc}
a_{22} & -a_{12} \\
-a_{21} & a_{22}
\end{array}\right)\left(\begin{array}{c}
s_{t} \\
f_{t}
\end{array}\right)=\left(\begin{array}{c}
e_{t} \\
n_{t}
\end{array}\right) .
$$

Hence, $\left(1,-a_{22} a_{21}^{-1}\right)^{\prime}$ must be the co-integrating vector and $n_{t}$ represents the error correction term. Note that the efficient rate is often assumed to follow a geometrical Brownian motion in continuous time, which is consistent with $e_{t}$ being non-stationary. More importantly, if it is possible to estimate $e_{t}$ based on $s_{t}$ and $f_{t}$ then the various assumptions on efficient rate can be verified. The next section introduces a new test of cointegration which can be used to estimate $e_{t}$ and $n_{t}$.

\section{A NEW COINTEGRATION TEST}

This section proposes a new test of cointegration that takes advantage of non-normality. The test is inspired by the recent development in Independent Component Analysis (ICA). Although this section provides an overview of ICA, it is not a comprehensive survey on the subject given the space constraint. For more in depth introductions and latest developments, see Hérault and Ans (1984), Hérault et al. (1985), Hyvärinen et al. (2001), Common and Jutten (2010) and Common (1984).

Consider the following linear equation:

$$
\mathbf{y}_{t}=A \mathbf{x}_{t}
$$


where $\mathbf{y}_{t}=\left(y_{1 t}, . ., y_{k t}\right)^{\prime}$ is a $k \times 1$ vector, $A$ is a $k \times k$ real matrix and $\mathbf{x}_{t}=\left(x_{1 t}, \ldots, x_{k t}\right)^{\prime}$ is a $k \times 1$ vector of independent random variables with no more than one normal random variate. Moreover, $\mathbb{E}\left(\mathbf{x}_{t} \mathbf{x}_{t}^{\prime}\right)=\Omega_{t}$ exists for all $t$.

Under these assumptions, the standard problem is to recover $\mathbf{x}_{t}$ based on the observed sample of $\mathbf{y}_{t}$ without any information on $A$ or $\mathbf{x}_{t}$. Recall that an important implication of most central limit theorems is that a weighted sum of a sequence of random variables converges in distribution to normality. This implies that an additional random variable in an finite sequence will, in a sense, make the sum of that sequence "closer" to normal in distribution. This important insight allows the recovery of individual $x_{i t}$ if no more than one $x_{i t}$ is normally distributed. In order to take advantage of this insight, consider the following optimisation problem:

$$
\begin{gathered}
\hat{b}_{i}=\underset{b_{i}}{\arg \max } G\left(b_{i}^{\prime} \mathbf{y}_{t}\right) \\
\text { s.t } b_{i}^{\prime} b_{i}=1
\end{gathered}
$$

where $b_{i}$ is a $k \times 1$ vector and $G\left(b_{i}^{\prime} \mathbf{y}_{t}\right): \mathbb{R}^{k} \rightarrow \mathbb{R}$ is an appropriate measure of non-normality (see Hyvärinen et al. (2001) and Common and Jutten (2010) for various choice of $G$ ). Let $z_{i t}=b_{i}^{\prime} \mathbf{y}_{t}$ and $q_{i}^{\prime}=b_{i}^{\prime} A$ then $z_{i t}=b_{i}^{\prime} \mathbf{y}_{t}=b_{i}^{\prime} A \mathbf{x}_{t}=q_{i}^{\prime} \mathbf{x}_{t}$. Note that for a given element in $\mathbf{y}_{t}, y_{i t}=a_{i} . x_{t}$, where $a_{i}$. denotes the $i^{t h}$ row of $A$. Thus, $y_{i t}$ is a linear combination of at least $k-1$ non-normal random variables. Following the intuition as explained before, $y_{i t}$ must be "closer" to normal in distribution than any element in $\mathbf{x}_{t}$. Hence, in order to maximise the "non-normality" of $\hat{z}_{i t}=\hat{b}_{i}^{\prime} \mathbf{y}_{t}, \hat{b}_{i}$ must be the vector such that $\hat{q}_{i}^{\prime}=\hat{b}_{i}^{\prime} A$ has exactly one non-zero element. In other words, $\hat{z}_{i t}=\hat{q}_{i j} x_{j t}$ where $\hat{q}_{i j}$ is the $j^{\text {th }}$ element in the vector $\hat{q}_{i}$, for some $j \leq k$. Thus, one of the unobserved components has been recovered subject to a scale factor $\hat{q}_{i j}$. For a formal proof of this result, see Hyvärinen et al. (2001). The same process can be repeated in order to recover the remaining unobserved variables by utilising the independence nature of $\mathbf{x}_{t}$. This can be achieved by solving the same optimisation problem with an additional constraint that the next solution must be orthogonal to the previous ones.

Under the assumption that $A$ has full rank, there will be exactly $k$ orthogonal vectors, such that $b_{i}^{\prime} b_{j}=0$ for all $i \neq j$. Hence, the vector $\hat{b}_{k}$ can be calculated without solving the additional optimisation problem. Instead, it is simply the vector satisfying $\hat{b}_{k}^{\prime} \hat{b}_{i}=0$ for $i \neq k$. An implication of this result is that at most one of the unobserved components can be normally distributed.

Following the linear system as defined in equation (3), redefine $\mathbf{x}_{t}=\left(\mathbf{x}_{X t}^{\prime}, \mathbf{x}_{I t}^{\prime}\right)^{\prime}$ such that $\mathbf{x}_{X t}=$ $\left(x_{1 t}, \ldots, x_{r t}\right)^{\prime} \sim I(0)$ and $\mathbf{x}_{I t}=\left(x_{r+1 t}, \ldots, x_{k t}\right)^{\prime} \sim I(1)$. Note that if $\mathbf{y}_{t}=A \mathbf{x}_{t}$ then $\Delta \mathbf{y}_{t}=A \Delta \mathbf{x}_{t}$ where $\Delta$ denotes the first difference operator. Since $\mathbf{x}_{t} \sim I(1)$, it implies $\Delta \mathbf{x}_{t}$ has finite second moment and therefore, $A^{-1}$ can be recovered subject to scaling and permutation by solving the following optimsation problem:

$$
\begin{aligned}
& \hat{B}=\underset{B \in U}{\arg \max } G\left(B \Delta \mathbf{y}_{t}\right) \\
& \text { s.t. } B^{\prime} B=\mathbf{I}
\end{aligned}
$$

where I denotes the identity matrix with the appropriate dimension. Let $\hat{b}_{i}$ denotes the $i^{\text {th }}$ row of $\hat{B}$ and define $\hat{\mathbf{z}}_{t}=\left(\hat{z}_{1 t}, \ldots, \hat{z}_{k t}\right)^{\prime}$, such that $\hat{z}_{i t}=\hat{b}_{i} \mathbf{y}_{t}=\hat{q}_{i j} x_{j t}$, for some $j=1, . ., k$. That is, $\hat{z}_{i t}$ is a scalar multiple of one of the components in $\mathbf{x}_{t}$. In addition, due to the constraint $\hat{B}^{\prime} \hat{B}=\mathbf{I}$, the matrix $\hat{B}$ has full rank, so each element in $\hat{\mathbf{z}}_{t}$ is a scalar multiple of an unique element in $\mathbf{x}_{t}$.

Since each element in $\hat{\mathbf{z}}_{t}$ is a scalar multiple of an unique element in $\mathbf{x}_{t}$, there are exactly $r$ stationary variables and $l=k-r$ non-stationary variables in $\hat{\mathbf{z}}_{t}$. Thus, the cointegrating vectors, if exist, are the rows in $\hat{B}$ that correspond to the stationary components in $\hat{\mathbf{z}}_{t}$. Therefore, the test of cointegration can be performed by testing the stationarity of each row of $\hat{B} \mathbf{y}_{t}$.

An advantage of this approach is that the test of cointegration is reduced to multiple tests for unit root. More importantly, the order of integration is not required before conducting this test. This can have empirical advantage over the Johansen's approach, as it allows different tests of unit root with each row of $\mathbf{z}_{t}$, and can subsequently enhance the test power by accommodating different characteristics of the underlying variables, such as seasonality and structural break. 


\section{EMPIRICAL RESULTS}

Following the model as defined in equation (1), it is possible to apply the proposed test of cointegration to determine the cointegrating vector between spot and forward rates, and subsequently estimate $e_{t}$ and $n_{t}$. This is particularly interesting because it is well known that the daily spot and forward rates are not normally distributed in either level or first difference. Thus, the proposed test can exploit the non-normality nature of the variables.

This section applies the proposed test of co-integration to examine the forward-spot relationship in the exchange rate between U.S. and Australia. It utilises five-days daily spot and forward exchange rates data from DataStream with the sample starting from 17 September 1999 to 19 September 2012. This gives a total 3394 observations.

Under the assumption that $s_{t}$ follows a unit root process, the model as defined in equation (1) implies

$$
E_{t}\left(s_{t+1}\right)=s_{t}=\frac{a_{11}}{a_{21}} f_{t}-\frac{|A|}{a_{21}} n_{t}
$$

where $E_{t}$ denotes the expectation operator conditional on information available up to time $t$. Hence, FRUH holds if $a_{11}=a_{21}$ and $n_{t}=0$ for all $t$ i.e. market is efficient. The implication of the first condition is that the cointegrating vector between $s_{t}$ and $f_{t}$ must be $(1,-1)^{\prime}$ and the second condition allows an examination on the source of market inefficiency.

In terms of the order of integration, both spot and forward rates contain unit roots in level but not in first difference. This is confirmed by three different unit root tests, namely, the augmented Dicky Fuller (DF) test in Dickey and Fuller (1979) and Said and Dickey (1984), the Phillip-Perron (PP) test in Phillips and Perron (1988) and the Kwiatkowski-Phillips-Schmidt-Shin (KPSS) test in Kwiatkowski et al. (1992). The test results are available upon request.

Solving the optimisation problem as defined in equation (5) using the spot and forward data gives:

$$
\hat{B}=\left(\begin{array}{cc}
1 & -1.0343 \\
0.595 & 0.256
\end{array}\right)
$$

Pre-multiplying $\hat{B}$ with $\left(s_{t}, f_{t}\right)^{\prime}$ and apply unit root tests to the resulting series gives:

$$
\left(\begin{array}{cc}
1 & -1.0343 \\
0.595 & 0.256
\end{array}\right)\left(\begin{array}{l}
s_{t} \\
f_{t}
\end{array}\right)=\left(\begin{array}{l}
\hat{q}_{11} n_{t} \\
\hat{q}_{21} e_{t}
\end{array}\right) \text {. }
$$

This reflects the fact that the first row of $\hat{B}$ is the co-integrating vector as $s_{t}-1.0343 f_{t} \sim I(0)$ based on unit root test results. The estimates of the two unobserved components, $\hat{q}_{11} n_{t}$ and $\hat{q}_{21} e_{t}$, can be found in Figure 1. Interestingly, the non-stationary component resembles the dynamics in both spot and forward rates. The stationary component, however, exhibits completely different type of dynamics. Following from the standard assumptions in the literature, the non-stationary component can be considered as estimates for $e_{t}$, the efficient exchange rate, and the stationary component, $n_{t}$, can be considered as the "noise" induced by market behaviour and other external influences.

In order to compare the dynamic structures between $e_{t}$ and $n_{t}$ as well as the original spot and forward exchange rates, consider the following $\operatorname{ARMA}(p, q)-\operatorname{GARCH}(r, s)$ model:

$$
\begin{aligned}
\Phi(L)\left(y_{t}-\mu\right) & =\Theta(L) \varepsilon_{t} \\
\varepsilon_{t} & =\eta_{t} \sqrt{h_{t}} \\
h_{t} & =\omega+\sum_{i=1}^{r} \alpha_{i} \varepsilon_{t-i}^{2}+\sum_{i=1}^{s} \beta_{i} h_{t-i}
\end{aligned}
$$

where $\Phi(L)=1-\sum_{i=1}^{p} \phi_{i} L^{i}$ and $\Theta(L)=1+\sum_{i=1}^{q} \theta_{i} L^{i}$ with $L$ denotes the lag operator such that $L y_{t}=y_{t-1}$. It is assumed that $\Phi(L)$ and $\Theta(L)$ has no common or unit root. The structural and statistical properties of this model have been studied extensively in the literature, see for example, Ling and McAleer (2003). 

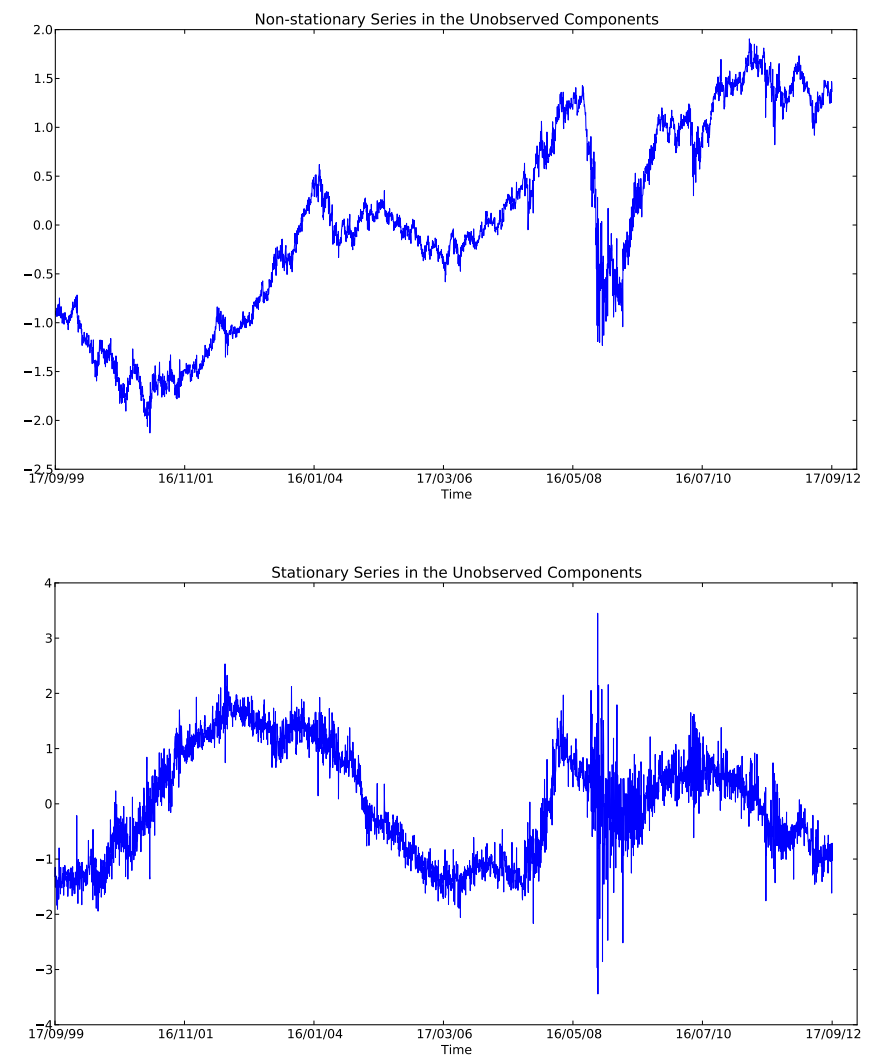

Figure 1. Unobserved Components

Following the spot-forward rate model as defined in equation (1), it implies that

$$
\left(\begin{array}{c}
\Delta s_{t} \\
\Delta f_{t}
\end{array}\right)=A\left(\begin{array}{c}
\Delta e_{t} \\
\Delta n_{t}
\end{array}\right)
$$

Note that the vectors on both sides of the equation are $I(0)$, this implies the parameters of the ARMA-GARCH model as defined in equation (7) can be consistently estimated via Quasi Maximum Likelihood Estimator (QMLE). For further details, see Ling and McAleer (2003).

The lag orders for the conditional mean and variance are determined by Schwarz-Bayesian Information Criterion (SBIC). Table 1 contains the estimation results for the four series.

As shown in Table 1, the dynamics between the observed and the unobserved are quite different. Interestingly, the best fit model for forward rate required a higher order lag in the conditional mean while the best fit model for the efficient rate, $e_{t}$, required a higher order lag in the conditional variance. More importantly, since both spot and forward rates are linear combination of $e_{t}$ and $n_{t}$, it means that the parameter estimates of spot and forward rates should be interpreted with cautions. This is due to the fact that aggregating a series of GARCH processes does not produce a GARCH process. This implies that the standard GARCH specification is misspecified for spot and forward rates, which casts doubts on the reliability on the parameter estimates for these two series.

Another interesting observation is that the standardised residuals did not seem to be normally distributed for all four series, as shown in Table 2. If the interpretation of efficient rate and market noise was valid in this case, it implied that neither the efficient rates nor the market noise was normally or identically distributed. This raises some interesting questions about the assumption of normality when modelling high frequency financial time series. Moreover, the best fit model for $e_{t}$ exhibited different dynamics than those implied by the standard Geometrical Brownian Motion (GBH). Thus, the application of GBH might also require further revision if the same empirical evidence found to be common among other financial time series. 
Table 1. Parameters Estimates for the Observed and Unobserved Series

\begin{tabular}{c|cccc}
\hline & $\Delta s_{t}$ & $\Delta f_{t}$ & $\Delta e_{t}$ & $\Delta n_{t}$ \\
\hline$\mu$ & 0.024 & -0.005 & -0.114 & -0.039 \\
& $(1.903)$ & $(-46.954)$ & $(-2.162)$ & $(-0.623)$ \\
$\phi_{1}$ & -0.206 & 0.342 & $1.18 \mathrm{e}-4$ & -0.048 \\
& $(-0.871)$ & $(23.498)$ & $(0.373)$ & $(-2.136)$ \\
$\phi_{2}$ & & 0.656 & & \\
& & $(28.545)$ & & \\
$\theta_{1}$ & 0.225 & -0.389 & -0.558 & -0.848 \\
& $(0.942)$ & $(-22.350)$ & $(-41.033)$ & $(-67.741)$ \\
$\theta_{2}$ & & -0.614 & & \\
& & $(-18.273)$ & & \\
$\omega$ & 0.007 & 0.008 & 1.992 & 15.600 \\
& $(3.447)$ & $(3.244)$ & $(3.969)$ & $(3.358)$ \\
$\alpha_{1}$ & 0.057 & 0.060 & 0.176 & 0.076 \\
& $(5.122)$ & $(5.773)$ & $(6.407)$ & $(4.831)$ \\
$\beta_{1}$ & 0.933 & 0.927 & 0.347 & 0.906 \\
& $(89.891)$ & $(95.126)$ & $(2.194)$ & $(53.271)$ \\
$\beta_{2}$ & & & 0.032 & \\
& & & $(0.252)$ & \\
$\beta_{3}$ & & & 0.418 & \\
& & & $(4.781)$ & \\
\hline
\end{tabular}

Table 2. Descriptive Statistics of the Standardised Residuals

\begin{tabular}{c|ccccc}
\hline & Mean & Deviation & Skewness & Kurtosis & Jarque-Bera Statistics \\
\hline$\Delta s_{t}$ & -0.014 & 0.998 & -0.441 & 4.740 & $537.820^{* * *}$ \\
$\Delta f_{t}$ & -0.011 & 0.996 & -0.378 & 3.994 & $220.547^{* * *}$ \\
$\Delta e_{t}$ & 0.008 & 0.999 & 0.416 & 4.786 & $480.431^{* * *}$ \\
$\Delta n_{t}$ & 0.011 & 0.998 & 0.245 & 6.461 & $702.206^{* * *}$ \\
\hline
\end{tabular}

\section{CONCLUSION}

This paper presented a simple model of spot-forward exchange rates and a new method of estimating cointegrating relationship that takes advantage of non-normality. The new method allows the identification and estimation of unobserved efficient exchange rate and market noise. By applying the proposed method to daily U.S. and Australia exchange rate data, the paper showed that the two exchange rates are cointegrated with the cointegrating vector consistent with the Forward Rate Unbiasedness Hypothesis. Moreover, both efficient exchange rate and market noise contained GARCH errors with different lag orders. This implied that the parameter estimates of GARCH models in the original spot and forward rates might be biased. Furthermore, the standardised residuals from both series were non-normal and thus, it provided some evidence against the assumption of normality when modelling financial time series data. More specifically, the assumption that the market noise is a normally and independent distributed random variate might not be appropriate.

Obviously, the empirical finding of this paper had been limited to one particular time series and therefore, the results cannot be generalised without further analysis on broader set of financial time series. However, the intention of this paper was not to challenge the standard practices in the literature, but rather, to demonstrate the usefulness of independent component analysis in analysing economic and financial time series. Specifically, this paper demonstrated that non-normality can be useful in identifying cointegration rank as well as identifying the unobserved factors governing the observed time series.

\section{ACKNOWLEDGEMENT}

The earlier version of this paper had been presented at the $23^{\text {th }}$ New Zealand Econometrics Study Group (NZESG) meeting. The author would like to thank Peter Phillips and Weshah Razzak for insightful discussion as well as the participants of the $23^{t h}$ NZESG meeting. The financial support from the Australian Research Council and the Bankwest Curtin Economic Centre are gratefully acknowledged. 
F. Chan, Identifying Efficient Exchange Rate Dynamics from Noisy Data

\section{REFERENCES}

Common, P. (1984). Independent component analysis - a new concept. Signal Processing 36, 287 - 314.

Common, P. and C. Jutten (Eds.) (2010). Handbook of Blind Source Separation. Academic Press.

Dickey, D. and W. Fuller (1979). Distributions of the estimators for autoregressive time series with a unit root. Journal of the American Statistical Association 74, 427-431.

Hérault, J. and B. Ans (1984). Circuits neuronaux à synapses modifiables: décodage de messages composites par apprentissage non supervisé. C.-R. de l'Académie des Sciences 299, 525-528.

Hérault, J., C. Jutten, and B. Ans (1985). Détection de grandeurs primitives dans un message composite par une architecture de calcul neuromimétique en apprentissage non supervisé. In Actes du Xème colloque GRETSI, pp. 1017-1022.

Hyvärinen, A., J. Karhunen, and E. Oja (2001). Independent Component Analysis. John Wiley \& Sons, Inc.

Johansen, S. (1988). Statistical analysis of cointegration vectors. Journal of Economic Dynamics and Control 12, 231-254.

Kwiatkowski, D., P. Phillips, P. Schmidt, and Y. Shin (1992). Testing the null hypothesis of stationarity against the alternative of a unit root: How sure are we that economic time series have a unit root? Journal of Econometrics 54, 159-178.

Ling, S. and M. McAleer (2003). Asymptotic theory for a Vector ARMA-GARCH model. Econometric Theory 19, 278-308.

Liuntel, K. and K. Paudyal (1998). Common stochastic trends between forward and spot exchange rate. Journal of International Monday and Finance 11, 366-381.

Mark, N. C. (1990). Real and nominal exchange rates in the long run" an empirical investigation. Journal of International Economics 28, 115-136.

Naka, A. and G. Whitney (1995). The unbiased forward rate hypothesis re-examined. Journal of International Money and Finance 14, 857-867.

Phillips, P. and P. Perron (1988). Testing for a unit root in time series regression. Biometrika 75, 335-346.

Said, S. and D. Dickey (1984). Testing for unit roots in autoregressive-moving average models of unknown order. Biometrika 71, 599-607.

Zivot, E. (2000). Cointegration and forward and spot exchange rate regressions. Journal of International Money and Finance 19, 785-812. 